\title{
STABILITY FOR RETARDED FUNCTIONAL DIFFERENTIAL EQUATIONS
}

\author{
M. Federson ${ }^{1}$ and $\check{\text { S. Schwabik }}{ }^{2}$
}

UDC 517.9

\begin{abstract}
It is known that retarded functional differential equations can be regarded as Banach-space-valued generalized ordinary differential equations (GODEs). In this paper, some stability concepts for retarded functional differential equations are introduced and they are discussed using known stability results for GODEs. Then the equivalence of the different concepts of stabilities considered here are proved and converse Lyapunov theorems for a very wide class of retarded functional differential equations are obtained by means of the correspondence of this class of equations with GODEs.
\end{abstract}

\section{Notation}

Let $X$ be a Banach space and let $I \subset \mathbb{R}$ be an interval of the real line.

We denote by $G(I, X)$ the space of locally regulated functions $f: I \rightarrow X$, i.e., for every compact interval $[a, b] \subset I$, the lateral limits $f(t+)=\lim _{\rho \rightarrow 0+} f(t+\rho), t \in[a, b)$, and $f(t-)=\lim _{\rho \rightarrow 0-} f(t+\rho), t \in(a, b]$, exist and are finite. If $I=[a, b]$, we write $G([a, b], X)$, which is a Banach space when endowed with the usual supremum norm. In $G(I, X)$, we consider the topology of locally uniform convergence. By $G^{-}(I, X)$, we mean the subspace of $G(I, X)$ of left continuous functions for which $f(t-)=\lim _{\rho \rightarrow 0-} f(t+\rho)=f(t), t \in I$, except for the left endpoint of $I$.

We denote by $B V(I, X)$ the space of functions $f: I \rightarrow X$ that are locally of bounded variation, i.e., for every compact interval $[a, b] \subset I$, the restriction $\left.f\right|_{[a, b]}$ of $f$ to $[a, b]$ is of bounded variation. In $B V([a, b], X)$, we consider the variation norm given by $\|f\|=\|f(a)\|+\operatorname{var}_{a}^{b} f$, where $\operatorname{var}_{a}^{b} f$ stands for the variation of $f$ in the interval $[a, b]$. Then $B V([a, b], X)$ is a Banach space and $B V([a, b], X) \subset G([a, b], X)$. If $f \in B V(I, X)$ is also left continuous $\left(f \in B V(I, X) \cap G^{-}(I, X)\right)$, we write $f \in B V^{-}(I, X)$.

We write $C(I, X)$ to denote the space of continuous functions $f: I \rightarrow X$. We consider the Banach space $C([a, b], X)$ equipped with the usual supremum norm, and in $C(I, X)$ we consider the topology of locally uniform convergence.

It is clear that $C(I, X) \subset G^{-}(I, X)$ and $B V^{-}(I, X) \subset G^{-}(I, X)$.

To simplify our considerations, we restrict ourselves to the case of left continuous functions everywhere when some discontinuities can occur.

\section{Retarded Functional Differential Equations}

Let us consider the initial-value problem for a retarded functional differential equation:

$$
\begin{gathered}
\dot{y}(t)=f\left(y_{t}, t\right), \\
y_{t_{0}}=\phi,
\end{gathered}
$$

\footnotetext{
${ }^{1}$ Institute of Mathematics Sciences and Computer Science, University of São Paulo, São Carlos, Brazil.

${ }^{2}$ Mathematical Institute, Academy of Sciences of Czech Republic, Prague, Czech Republic.
}

Published in Ukrains'kyi Matematychnyi Zhurnal, Vol. 60, No. 1, pp. 107-126, January, 2008. Original article submitted October 15, 2007. 
where $\phi \in G^{-}\left([-r, 0], \mathbb{R}^{n}\right), r \geq 0$, and $f(\phi, t)$ maps an open subset $\Omega$ of $G^{-}\left([-r, 0], \mathbb{R}^{n}\right) \times\left[t_{0},+\infty\right)$ to $\mathbb{R}^{n}$. Given a function $y:\left[t_{0}-r,+\infty\right) \rightarrow \mathbb{R}^{n}$, we consider $y_{t}:[-r, 0] \rightarrow \mathbb{R}^{n}$ defined, as usual, by

$$
y_{t}(\theta)=y(t+\theta), \quad \theta \in[-r, 0], \quad t \in\left[t_{0},+\infty\right)
$$

Let us recall the concept of a solution of problem (1.1).

Definition 1.1. Let $\sigma>0$. A function $y \in G^{-}\left(\left[t_{0}-r, t_{0}+\sigma\right], \mathbb{R}^{n}\right)$ such that $\left(y_{t}, t\right) \in G^{-}\left([-r, 0], \mathbb{R}^{n}\right) \times$ $\left[t_{0}, t_{0}+\sigma\right]$ for all $t \in\left[t_{0}, t_{0}+\sigma\right], y_{t_{0}}=\phi$, and

$$
\dot{y}(t)=f\left(y_{t}, t\right)
$$

for almost all $t \in\left[t_{0}, t_{0}+\sigma\right]$ is called a (local) solution of (1.1) in $\left[t_{0}, t_{0}+\sigma\right]$ (or sometimes also in $\left.\left[t_{0}-r, t_{0}+\sigma\right]\right)$ with initial condition $\left(\phi, t_{0}\right)$.

System (1.1) is known to be equivalent to the "integral" equation

$$
\begin{gathered}
y(t)=y\left(t_{0}\right)+\int_{t_{0}}^{t} f\left(y_{s}, s\right) d s, \quad t \in\left[t_{0},+\infty\right), \\
y_{t_{0}}=\phi,
\end{gathered}
$$

when the integral exists in the Lebesgue sense (cf. [1]). In fact, we will use (1.2) for the concept of the initial-value problem (1.1). This makes it clear that if a solution $y$ is defined on some interval $\left[t_{0}, t_{0}+\sigma\right]$ with $\sigma>0$, then $y$, being an indefinite integral of a Lebesgue integrable function, is necessarily absolutely continuous on $\left[t_{0}, t_{0}+\sigma\right]$ (we write $\left.y \in A C\left(\left[t_{0}, t_{0}+\sigma\right], \mathbb{R}^{n}\right)\right)$.

Let $G_{1} \subset G^{-}\left(\left[t_{0}-r,+\infty\right), \mathbb{R}^{n}\right)$ with the following property: if $y=y(t), t \in\left[t_{0}-r,+\infty\right)$, is an element of $G_{1}$ and $\bar{t} \in\left[t_{0}-r,+\infty\right)$, then $\bar{y}$ given by

$$
\bar{y}(t)= \begin{cases}y(t), & t_{0}-r \leq t \leq \bar{t} \\ y(\bar{t}), & \bar{t}<t<+\infty\end{cases}
$$

also belongs to $G_{1}$.

Let $L_{1}(I, X)$ denote the space of locally Bochner integrable functions $f: I \rightarrow X$ integrable in every compact of $I$, where $I \subset \mathbb{R}$ is an interval and $X$ is a Banach space. If $X$ is finite-dimensional, then we have the Lebesgue integral in mind.

Let $|\cdot|$ be a norm in $\mathbb{R}^{n}$.

We consider $f(\phi, t): G^{-}\left([-r, 0], \mathbb{R}^{n}\right) \times\left[t_{0},+\infty\right) \rightarrow \mathbb{R}^{n}$, the right-hand side of the differential equation in (1.1) such that the mapping $t \mapsto f\left(y_{t}, t\right)$ belongs to $L_{1}\left(\left[t_{0},+\infty\right), \mathbb{R}^{n}\right)$ for $y \in G_{1}$ and the following conditions are satisfied:

(A) there is $M \in L_{1}\left(\left[t_{0},+\infty\right), \mathbb{R}\right)$ such that, for all $x \in G_{1}$ and $u_{1}, u_{2} \in\left[t_{0},+\infty\right)$,

$$
\left|\int_{u_{1}}^{u_{2}} f\left(x_{s}, s\right) d s\right| \leq \int_{u_{1}}^{u_{2}} M(s) d s
$$


(B) there is $L \in L_{1}\left(\left[t_{0},+\infty\right), \mathbb{R}\right)$ such that, for all $x, y \in G_{1}$ and $u_{1}, u_{2} \in\left[t_{0},+\infty\right)$,

$$
\left|\int_{u_{1}}^{u_{2}}\left[f\left(x_{s}, s\right)-f\left(y_{s}, s\right)\right] d s\right| \leq \int_{u_{1}}^{u_{2}} L(s)\left\|x_{s}-y_{s}\right\| d s
$$

the norm on the right-hand side is the norm in $G^{-}\left([-r, 0], \mathbb{R}^{n}\right)$ given by $\|\phi\|=\sup _{t \in[-r, 0]}|\phi(t)|$ for $\phi \in G^{-}\left([-r, 0], \mathbb{R}^{n}\right)$.

Of course, the functions $M$ and $L$ above depend on the choice of $t_{0}$.

If $f(0, t)=0$ for every $t \in \mathbb{R}$, then $y \equiv 0$ is a solution of (1.1). The next definitions concern stability concepts for the solution $y \equiv 0$ of (1.1). The following three definitions are the classical definitions for Lyapunov stability, uniform (Lyapunov) stability, and uniform asymptotic stability of the trivial solution of (1.1) (see, e.g., [1]):

Definition 1.2. The trivial solution of system (1.1) is called (Lyapunov) stable if, for every $\varepsilon>0$, there exists $\delta=\delta\left(\varepsilon, t_{0}\right)>0$ such that if $\phi \in G^{-}\left([-r, 0], \mathbb{R}^{n}\right)$ and $\bar{y}:[\gamma, v] \rightarrow \mathbb{R}^{n}$ with $[\gamma, v] \subset\left[t_{0}-r,+\infty\right)$ and $[\gamma, v] \ni t_{0}$ is a solution of (1.1) such that $\bar{y}_{t_{0}}=\phi$ and

$$
\|\phi\|<\delta
$$

then

$$
\left\|\bar{y}_{t}\left(t_{0}, \phi\right)\right\|<\varepsilon, \quad t \in\left[t_{0}, v\right]
$$

Definition 1.3. The trivial solution of system (1.1) is called uniformly stable if the number $\delta$ in Definition 1.2 is independent of $t_{0}$.

Definition 1.4. The solution $y \equiv 0$ of (1.1) is called uniformly asymptotically stable if there exists $\delta_{0}>0$ and, for every $\varepsilon>0$, there exists $T=T\left(\varepsilon, \delta_{0}\right) \geq 0$ such that if $\phi \in G^{-}\left([-r, 0], \mathbb{R}^{n}\right)$ and $\bar{y}:[\gamma, v] \rightarrow \mathbb{R}^{n}$ with $[\gamma, v] \subset\left[t_{0}-r,+\infty\right)$ and $[\gamma, v] \ni t_{0}$ is a solution of (1.1) such that $\bar{y}_{t_{0}}=\phi$ and

$$
\|\phi\|<\delta_{0}
$$

then

$$
\left\|\bar{y}_{t}\left(t_{0}, \phi\right)\right\|<\varepsilon, \quad t \in[\gamma, v] \cap[\gamma+T,+\infty) .
$$

The next definition of stability of the solution $y \equiv 0$ of (1.1) is borrowed from [2].

Definition 1.5. The solution $y \equiv 0$ of (1.1) is said to be integrally stable if, for every $\varepsilon>0$, there is $\delta=\delta(\varepsilon)>0$ such that if $\phi \in G^{-}\left([-r, 0], \mathbb{R}^{n}\right),\|\phi\|<\delta$, and $p \in L_{1}\left(\left[t_{0}, t_{1}\right], \mathbb{R}^{n}\right)$ with $\int_{t_{0}}^{t_{1}}|p(s)| d s<\delta$, then

$$
\left|y\left(t ; t_{0}, \phi\right)\right|<\varepsilon \quad \text { for every } t \in\left[t_{0}, t_{1}\right] \text {, }
$$


where $y\left(t ; t_{0}, \phi\right)$ is a solution of the perturbed equation

$$
\begin{gathered}
\dot{y}(t)=f\left(y_{t}, t\right)+p(t), \\
y_{t_{0}}=\phi .
\end{gathered}
$$

The solution of Eq. (1.3) has to be interpreted as a solution of the "integral" equation

$$
\begin{gathered}
y(t)=y\left(t_{0}\right)+\int_{t_{0}}^{t} f\left(y_{s}, s\right) d s+\int_{t_{0}}^{t} p(s) d s, \\
y_{t_{0}}=\phi,
\end{gathered}
$$

where the integral is considered in the Lebesgue sense. The solution of (1.3), when it exists, is absolutely continuous on $\left[t_{0}, t_{1}\right]$ (i.e., $\left.y\left(\cdot ; t_{0}, \phi\right) \in A C\left(\left[t_{0}, t_{1}\right], \mathbb{R}^{n}\right)\right)$.

We now introduce a concept of stability of the trivial solution of (1.1) that generalizes Definition 1.5 and will be essential to our purposes.

Definition 1.6. The solution $y \equiv 0$ of (1.1) is said to be variationally stable if, for every $\varepsilon>0$, there is a $\delta=\delta(\varepsilon)>0$ such that if $\phi \in G^{-}\left([-r, 0], \mathbb{R}^{n}\right),\|\phi\|<\delta$, and $P \in B V^{-}\left(\left[t_{0}, t_{1}\right], \mathbb{R}^{n}\right)$ with $\operatorname{var}_{t_{0}}^{t_{1}} P<\delta$, then

$$
\left|y\left(t ; t_{0}, \phi\right)\right|<\varepsilon \quad \text { for every } t \in\left[t_{0}, t_{1}\right],
$$

where $y\left(t ; t_{0}, \phi\right)$ is a solution of

$$
\begin{gathered}
y(t)=y\left(t_{0}\right)+\int_{t_{0}}^{t} f\left(y_{s}, s\right) d s+P(t)-P\left(t_{0}\right), \quad t \in\left[t_{0}, t_{1}\right], \\
y_{t_{0}}=\phi .
\end{gathered}
$$

It can be seen immediately that the solution $y$ of (1.5) is of bounded variation and left continuous, i.e., $y \in B V^{-}\left(\left[t_{0}, t_{1}\right], \mathbb{R}^{n}\right) \subset G^{-}\left(\left[t_{0}, t_{1}\right], \mathbb{R}^{n}\right)$.

Note that (1.4) is a particular case of (1.5) for $P(t)=\int_{t_{0}}^{t} p(s) d s, t \geq t_{0}$. If $p \in L_{1}\left(\left[t_{0}, t_{1}\right], \mathbb{R}^{n}\right)$, then we have $P \in A C\left(\left[t_{0}, t_{1}\right], \mathbb{R}^{n}\right) \subset B V^{-}\left(\left[t_{0}, t_{1}\right], \mathbb{R}^{n}\right)$, the derivative $\dot{P}(s)=\frac{d P}{d s}$ exists almost everywhere in $\left[t_{0}, t_{1}\right]$, and

$$
\operatorname{var}_{t_{0}}^{t_{1}} P=\int_{t_{0}}^{t_{1}}|\dot{P}(s)| d s=\int_{t_{0}}^{t_{1}}|p(s)| d s .
$$

Having this in mind, we can easily see that the variational stability of the trivial solution of (1.1) is a more general concept than that of integral stability. Therefore, we consider only the variational stability. 
Definition 1.7. The solution $y \equiv 0$ of (1.1) is called variationally attracting if there is $\widetilde{\delta}>0$ and, for every $\varepsilon>0$, there exist $T=T(\varepsilon) \geq 0$ and $\rho=\rho(\varepsilon)>0$ such that if

$$
\|\phi\|<\widetilde{\delta} \quad \text { and } \quad \operatorname{var}_{t_{0}}^{t_{1}} P<\rho
$$

with $P \in B V^{-}\left(\left[t_{0}, t_{1}\right], \mathbb{R}^{n}\right)$, then

$$
\left|y\left(t ; t_{0}, \phi\right)\right|<\varepsilon \quad \text { for all } t \geq t_{0}+T, \quad t \in\left[t_{0}, t_{1}\right]
$$

where $y\left(t ; t_{0}, \phi\right)$ is a solution of Eq. (1.5) satisfying $y_{t_{0}}=\phi$.

Definition 1.8. The solution $y \equiv 0$ of (1.1) is called variationally asymptotically stable if it is variationally stable and variationally attracting.

It is clear by definition that if the solution $y \equiv 0$ of (1.1) is variationally stable, then it is also Lyapunov stable. A similar statement is also true for asymptotic stabilities.

Maybe, the reader is wondering at this moment why Definitions 1.6 to 1.8 are presented for RFDEs. One reason is that stability with respect to permanently acting perturbations is of interest for technology. The second is a pragmatic one, since we have results on stability for GODEs at our disposal that can be used in this context. See $[3,4]$ and the development of the theory in the next section.

To the first reason we add that the perturbation in the case of integral stability can be large enough as long as its integral is small. One could also consider perturbations of the form $p\left(t, y, y_{t}\right)$ and the same technique would apply. However, the theory around would be more complicated technically. In the case of variational stability, we can think about the possibility of perturbing the original equation (1.1) by an integrable function plus a Dirac sum acting on a countable set and then interpret the solution appropriately. In this case, the solution is a left continuous function. It is clear that (1.5) can be interpreted as an equation with impulses acting at points of discontinuity of the function $P$ and described in the form given, e.g., in the book [5] and, of course, in numerous papers of the Kiev ODE group concentrated around these two personalities.

\section{GODE Corresponding to (1.5)}

Let $X$ be a Banach space and consider $\Omega \subset X \times \mathbb{R}$. Assume that $G: \Omega \rightarrow X$ is a given $X$-valued function with $G(x, t)$ defined for every $(x, t) \in \Omega$.

Having the concept of Kurzweil integrability in mind (see, e.g., [3, 6, 7] or [4]), we now present the concept of generalized differential equation.

Definition 2.1. A function $x:[\alpha, \beta] \rightarrow X$ is called a solution of the generalized ordinary differential equation

$$
\frac{d x}{d \tau}=D G(x, t)
$$

on the interval $[\alpha, \beta] \subset \mathbb{R}$ if $(x(t), t) \in \Omega$ for all $t \in[\alpha, \beta]$ and if the equality

$$
x(v)-x(\gamma)=\int_{\gamma}^{v} D G(x(\tau), t)
$$

holds for every $\gamma, v \in[\alpha, \beta]$, where the integral is considered in the sense of Kurzweil. 
Let us mention that the theory of generalized ordinary differential equations presented, e.g., in [7] is for the case where $X=\mathbb{R}^{n}$, but it is easy to check that all basic results also hold in the case of a Banach space.

Given an initial condition $\left(z_{0}, t_{0}\right) \in \Omega$, the following definition of the solution of the initial-value problem for Eq. (2.1) will be used.

Definition 2.2. A function $x:[\alpha, \beta] \rightarrow X$ is a solution of the generalized ordinary differential equation (2.1) with initial condition $x\left(t_{0}\right)=z_{0}$ on the interval $[\alpha, \beta] \subset \mathbb{R}$ if $t_{0} \in[\alpha, \beta],(x(t), t) \in \Omega$ for all $t \in[\alpha, \beta]$, and the equality

$$
x(v)-z_{0}=\int_{t_{0}}^{v} D G(x(\tau), t)
$$

holds for every $v \in[\alpha, \beta]$.

We now consider $\Omega=G_{1} \times\left[t_{0},+\infty\right)$ and define a special class of functions $F: \Omega \rightarrow X$.

Definition 2.3. We say that a function $G: \Omega \rightarrow X$ belongs to the class $\mathcal{F}(\Omega, h)$ if there exists a nondecreasing left continuous function $h:\left[t_{0},+\infty\right) \rightarrow \mathbb{R}$ such that

$$
\left\|G\left(x, s_{2}\right)-G\left(x, s_{1}\right)\right\| \leq\left|h\left(s_{2}\right)-h\left(s_{1}\right)\right|
$$

for all $\left(x, s_{2}\right),\left(x, s_{1}\right) \in \Omega$ and

$$
\left\|G\left(x, s_{2}\right)-G\left(x, s_{1}\right)-G\left(y, s_{2}\right)+G\left(y, s_{1}\right)\right\| \leq\|x-y\|\left|h\left(s_{2}\right)-h\left(s_{1}\right)\right|
$$

for all $\left(x, s_{2}\right),\left(x, s_{1}\right),\left(y, s_{2}\right),\left(y, s_{1}\right) \in \Omega$.

Suppose that $f(\phi, t): G_{1} \times\left[t_{0},+\infty\right) \rightarrow \mathbb{R}^{n}$ is such that, for every $y \in G_{1}$, the mapping $t \mapsto f\left(y_{t}, t\right)$ belongs to $L_{1}\left(\left[t_{0},+\infty\right), \mathbb{R}^{n}\right)$ and $f$ satisfies conditions (A) and (B).

Assume further that $P \in B V^{-}\left(\left[t_{0},+\infty\right), \mathbb{R}^{n}\right)$.

For $y \in G_{1}$ and $t \in\left[t_{0}-r,+\infty\right)$, we define

$$
F(y, t)(\vartheta)= \begin{cases}0, & t_{0}-r \leq \vartheta \leq t_{0} \quad \text { or } \quad t_{0}-r \leq t \leq t_{0}, \\ \int_{t_{0}}^{\vartheta} f\left(y_{s}, s\right) d s, & t_{0} \leq \vartheta \leq t<+\infty \\ \int_{t_{0}}^{t} f\left(y_{s}, s\right) d s, & t_{0} \leq t \leq \vartheta<+\infty\end{cases}
$$

and for $t \in\left[t_{0}-r,+\infty\right)$ we set

$$
\bar{P}(t)(\vartheta)= \begin{cases}0, & t_{0}-r \leq \vartheta \leq t_{0} \quad \text { or } \quad t_{0}-r \leq t \leq t_{0}, \\ P(\vartheta)-P\left(t_{0}\right), & t_{0} \leq \vartheta \leq t<+\infty, \\ P(t)-P\left(t_{0}\right), & t_{0} \leq t \leq \vartheta<+\infty .\end{cases}
$$


Then

$$
G(y, t)=F(y, t)+\bar{P}(t)
$$

defines an element $G(y, t)$ of $G^{-}\left(\left[t_{0}-r,+\infty\right), \mathbb{R}^{n}\right)$, and $G(y, t)(\vartheta) \in \mathbb{R}^{n}$ is the value of $G(y, t)$ at a point $\vartheta \in\left[t_{0}-r,+\infty\right)$, i.e.,

$$
G: G_{1} \times\left[t_{0}-r,+\infty\right) \rightarrow G^{-}\left(\left[t_{0}-r,+\infty\right), \mathbb{R}^{n}\right) .
$$

The idea to construct the right-hand side of a GODE that corresponds to a functional differential equation of the form (1.1) is due to Imaz, Oliva, and Vorel $[8,9]$.

Let $h:\left[t_{0},+\infty\right) \rightarrow \mathbb{R}$ be defined by

$$
h(t)=\int_{t_{0}}^{t}[M(s)+L(s)] d s+\operatorname{var}_{t_{0}}^{t} P, \quad t \in\left[t_{0},+\infty\right) .
$$

Then the function $h$ is left continuous and nondecreasing, since $M, L:\left[t_{0},+\infty\right) \rightarrow \mathbb{R}$ are nonnegative a.e. and $P \in B V^{-}\left(\left[t_{0},+\infty\right), \mathbb{R}^{n}\right)$.

Under the given assumptions, it is a matter of routine to prove that the function $G$ given by (2.8) belongs to the class $\mathcal{F}(\Omega, h)$, where $\Omega=G_{1} \times\left[t_{0},+\infty\right)$ (see, e.g., [6]).

Consider $G$ given by (2.8). If $[\alpha, \beta] \subset\left[t_{0},+\infty\right)$ and $x:[\alpha, \beta] \rightarrow G^{-}\left(\left[t_{0}-r,+\infty\right), \mathbb{R}^{n}\right)$ is a solution of (2.1) in $[\alpha, \beta]$, then $x$ is of bounded variation in $[\alpha, \beta]$ and

$$
\operatorname{var}_{\alpha}^{\beta} x \leq h(\beta)-h(\alpha)<+\infty
$$

Moreover, every point in $[\alpha, \beta]$ at which the function $h$ is continuous is a point of continuity of the solution $x:[\alpha, \beta] \rightarrow G^{-}\left(\left[t_{0}-r,+\infty\right), \mathbb{R}^{n}\right)$, and we have

$$
x(\sigma+)-x(\sigma)=\lim _{s \rightarrow \sigma+} x(s)-x(\sigma)=G(x(\sigma), \sigma+)-G(x(\sigma), \sigma)
$$

for $\sigma \in[\alpha, \beta)$ and

$$
x(\sigma)-x(\sigma-)=x(\sigma)-\lim _{s \rightarrow \sigma-} x(s)=G(x(\sigma), \sigma)-G(x(\sigma), \sigma-)
$$

for $\sigma \in(\alpha, \beta]$, where $G(x, \sigma+)=\lim _{s \rightarrow \sigma+} G(x, s)$ for $\sigma \in[\alpha, \beta)$, and $G(x, \sigma-)=\lim _{s \rightarrow \sigma-} G(x, s)$ for $\sigma \in(\alpha, \beta]$. For a proof of these facts, the reader may want to consult, e.g., [7].

We now present a result on the existence of the integral involved in the definition of the solution of the generalized equation (2.1). This result is a particular case of Corollary 3.16 and Proposition 3.6, both from [7].

Lemma 2.1. Let $G \in \mathcal{F}(\Omega, h)$. Suppose that $x:[\alpha, \beta] \rightarrow X,[\alpha, \beta] \subset\left[t_{0},+\infty\right)$, is of bounded variation in $[\alpha, \beta]$ and $(x(s), s) \in \Omega$ for every $s \in[\alpha, \beta]$. Then the integral $\int_{\alpha}^{\beta} D G(x(\tau), t)$ exists and the function $s \mapsto \int_{\alpha}^{s} D G(x(\tau), t) \in X$ is of bounded variation for all $s \in[\alpha, \beta]$.

The next result concerns the existence of a solution of (2.1) (see [6], Theorem 2.15). 
Theorem 2.1. Let $G: \Omega \rightarrow X$ be an element of the class $\mathcal{F}(\Omega, h)$, where the function $h$ is left continuous (i.e., $h(t-)=h(t), t \in(a,+\infty))$. Then, for every $\left(\widetilde{x}, t_{0}\right) \in \Omega$ such that, for $\widetilde{x}_{+}=\widetilde{x}+G\left(\widetilde{x}, t_{0}+\right)-G\left(\widetilde{x}, t_{0}\right)$, we have $\left(\widetilde{x}_{+}, t_{0}\right) \in \Omega$, there exists $\Delta>0$ such that, on the interval $\left[t_{0}, t_{0}+\Delta\right]$, there exists a unique solution $x:\left[t_{0}, t_{0}+\Delta\right] \rightarrow X$ of the generalized ordinary differential equation (2.1) for which $x\left(t_{0}\right)=\widetilde{x}$.

Consider the generalized equation (2.1). We now work with a specific initial-value problem for Eq. (2.3) with $G$ given by (2.8).

Let $\phi \in G^{-}\left([-r, 0], \mathbb{R}^{n}\right)$ and $\sigma>0$ be given. A function $x(t)$ defined on the interval $\left[t_{0}-r, t_{0}+\sigma\right]$ and taking values in $G^{-}\left(\left[t_{0}-r, t_{0}+\sigma\right], \mathbb{R}^{n}\right)$ is a (local) solution of the generalized ordinary differential equation (2.1) in the interval $\left[t_{0}, t_{0}+\sigma\right]$ (or in $\left.\left[t_{0}-r, t_{0}+\sigma\right]\right)$ with initial condition $x\left(t_{0}\right) \in G_{1}$ given for $\phi \in G^{-}\left([-r, 0], \mathbb{R}^{n}\right)$ by

$$
x\left(t_{0}\right)(\vartheta)=\left\{\begin{array}{lll}
\phi\left(\vartheta-t_{0}\right) & \text { for } & \vartheta \in\left[t_{0}-r, t_{0}\right], \\
x\left(t_{0}\right)\left(t_{0}\right) & \text { for } & \vartheta \in\left[t_{0}, t_{0}+\sigma\right]
\end{array}\right.
$$

if

$$
x(v)=x\left(t_{0}\right)+\int_{t_{0}}^{v} D G(x(\tau), t)
$$

for every $v \in\left[t_{0}, t_{0}+\sigma\right]$.

For a proof of the next result, see [6], Lemma 3.3.

Proposition 2.1. If $x(t)$ is a solution of (2.1) in the interval $\left[t_{0}, t_{0}+\sigma\right]$, then, for $v \in\left[t_{0}, t_{0}+\sigma\right]$, we have

$$
x(v)(\vartheta)=x(v)(v), \quad \vartheta \geq v, \quad \vartheta \in\left[t_{0}-r, t_{0}+\sigma\right],
$$

and

$$
x(v)(\vartheta)=x(\vartheta)(\vartheta), \quad v \geq \vartheta, \quad \vartheta \in\left[t_{0}-r, t_{0}+\sigma\right] .
$$

Left continuous regulated functions with the properties of Proposition 2.1 are candidates for considering them as solutions of the initial-value problem described above for (2.1).

The next result is the key to our approach to retarded functional differential equations by the theory of generalized differential equations. It states the correspondence between these equations by relating their solutions in a one-to-one manner. For a proof of it, see [6].

Proposition 2.2. The following assertions are true:

(i) Consider Eq.(1.5), where $f: G_{1} \times\left[t_{0}, t_{0}+\sigma\right] \rightarrow \mathbb{R}^{n}, t \mapsto f\left(y_{t}, t\right)$ is Lebesgue integrable on $\left[t_{0}, t_{0}+\sigma\right], P \in B V^{-}\left(\left[t_{0}, t_{0}+\sigma\right], \mathbb{R}^{n}\right)$, and conditions $(A)$ and $(B)$ are satisfied. Let $y(t)$ be a solution of problem (1.5) in the interval $\left[t_{0}, t_{0}+\sigma\right]$. Given $t \in\left[t_{0}-r, t_{0}+\sigma\right]$, let

$$
x(t)(\vartheta)= \begin{cases}y(\vartheta), & \vartheta \in\left[t_{0}-r, t\right], \\ y(t), & \vartheta \in\left[t, t_{0}+\sigma\right] .\end{cases}
$$

Then $x(t) \in G^{-}\left(\left[t_{0}-r, t_{0}+\sigma\right], \mathbb{R}^{n}\right)$ is a solution of $(2.1)$ in $\left[t_{0}-r, t_{0}+\sigma\right]$, where the right-hand side of (2.1) is given by (2.8). 
(ii) Conversely, let $G$ be given by (2.8) and let $x(t)$ be a solution of (2.1) in the interval $\left[t_{0}-r, t_{0}+\sigma\right]$ satisfying the initial condition

$$
x\left(t_{0}\right)(\vartheta)= \begin{cases}\phi\left(\vartheta-t_{0}\right), & t_{0}-r \leq \vartheta \leq t_{0}, \\ x\left(t_{0}\right)\left(t_{0}\right), & t_{0} \leq \vartheta \leq t_{0}+\sigma .\end{cases}
$$

For every $\vartheta \in\left[t_{0}-r, t_{0}+\sigma\right]$, define

$$
y(\vartheta)= \begin{cases}x\left(t_{0}\right)(\vartheta), & t_{0}-r \leq \vartheta \leq t_{0}, \\ x(\vartheta)(\vartheta), & t_{0} \leq \vartheta \leq t_{0}+\sigma .\end{cases}
$$

Then $y(\vartheta)$ is a solution of (1.5) in $\left[t_{0}-r, t_{0}+\sigma\right]$ and $y(\vartheta)=x\left(t_{0}+\sigma\right)(\vartheta)$ for all $\vartheta \in$ $\left[t_{0}-r, t_{0}+\sigma\right]$.

Proposition 2.2 gives a one-to-one correspondence between the solutions $y$ of (1.5) and the solutions $x$ of (2.1). Thus, given a solution $y$ of (1.5), we have an $x$ given by Proposition 2.2, (i), that satisfies Eq. (2.1). Therefore, taking $t_{0} \leq t_{1} \leq t_{2} \leq t_{0}+\sigma$, we get

$$
\begin{aligned}
\left\|x\left(t_{2}\right)-x\left(t_{1}\right)\right\| & =\sup _{\vartheta \in\left[t_{0}-r, t_{0}+\sigma\right]}\left|x\left(t_{2}\right)(\vartheta)-x\left(t_{1}\right)(\vartheta)\right| \\
& =\sup _{\vartheta \in\left[t_{0}, t_{0}+\sigma\right]}\left|x\left(t_{2}\right)(\vartheta)-x\left(t_{1}\right)(\vartheta)\right|=\sup _{\vartheta \in\left[t_{1}, t_{2}\right]}\left|y(\vartheta)-y\left(t_{1}\right)\right| \leq \operatorname{var}_{t_{1}}^{t_{2}} y,
\end{aligned}
$$

and taking $t_{0}<t_{1}<t_{2}<\ldots<t_{k}=t_{0}+\sigma$, we get

$$
\sum_{i=1}^{k}\left\|x\left(t_{i}\right)-x\left(t_{i-1}\right)\right\| \leq \sum_{i=1}^{k} \operatorname{var}_{t_{i-1}}^{t_{i}} y=\operatorname{var}_{t_{0}}^{t_{0}+\sigma} y .
$$

Hence,

$$
\operatorname{var}_{t_{0}}^{t_{0}+\sigma} x \leq \operatorname{var}_{t_{0}}^{t_{0}+\sigma} y
$$

It has to be noted that a solution $y$ of (1.5) is a function of bounded variation, and, therefore, the corresponding $x$ is also of bounded variation.

Conversely, if $G$ is given by (2.8) and $x(t)$ is a solution of (2.1) with initial condition (2.9), then it can be shown by the procedure above that $y$ given by Proposition 2.2, (ii), satisfies

$$
\operatorname{var}_{t_{0}}^{t_{0}+\sigma} y \leq \operatorname{var}_{t_{0}}^{t_{0}+\sigma} x<+\infty
$$

In this manner, we have the situation of a one-to-one correspondence between the solutions of (1.5) and (2.1), and their variations (in different spaces) are the same and finite.

Remark 2.1. Let us note that, in our paper [6], a similar approach to impulsive retarded functional equations was presented. Of course, the definition in this case is slightly more complicated by an additional term. The complication is technical only, and the reasoning of this note can be used similarly for this case, too. Again, the link between GODEs and classical systems with impulses as they are described in [5] is given in [7]. 


\section{Concepts of Stability for GODEs}

In this section, $\Omega=B_{c} \times\left[t_{0}-r, \infty\right)$, where $B_{c}=\{y \in X ;\|y\|<c\}, c>0$, and $X$ is any Banach space. Let $r \geq 0$. In the sequel, we assume that, for $F: \Omega \rightarrow X$, we have $F \in \mathcal{F}(\Omega, h)$ and $F(0, t)-F(0, s)=0$ for $t, s \in\left[t_{0}-r,+\infty\right)$. Then, for every $[\gamma, v] \subset\left[t_{0}-r,+\infty\right)$, we get

$$
\int_{\gamma}^{v} D F(0, t)=F(0, v)-F(0, \gamma)=0
$$

and, therefore, $x \equiv 0$ is a solution of the generalized equation

$$
\frac{d x}{d \tau}=D F(x, t)
$$

on $\left[t_{0}-r,+\infty\right)$.

If $F \in \mathcal{F}(\Omega, h)$ and $x:[\gamma, v] \rightarrow X$ is a solution of (3.1), where $[\gamma, v] \subset\left[t_{0}-r,+\infty\right)$, then $x$ is of bounded variation in $[\gamma, v]$. Thus, it is natural to measure the distance between two solutions by the variation norm.

The next stability concepts are based on the variation of solutions around $x \equiv 0$.

Definition 3.1. The solution $x \equiv 0$ of (3.1) is called variationally stable if, for every $\varepsilon>0$, there exists $\delta=\delta(\varepsilon)>0$ such that if $\bar{x}:[\gamma, v] \rightarrow B_{c}, t_{0}-r \leq \gamma<v<+\infty$, is a function of bounded variation on $[\gamma, v]$ such that

$$
\|\bar{x}(\gamma)\|<\delta
$$

and

$$
\operatorname{var}_{\gamma}^{v}\left(\bar{x}(s)-\int_{\gamma}^{s} D F(\bar{x}(\tau), t)\right)<\delta,
$$

then

$$
\|\bar{x}(t)\|<\varepsilon, \quad t \in[\gamma, v] .
$$

Definition 3.2. The solution $x \equiv 0$ of (3.1) is called variationally attracting if there exists $\delta_{0}>0$ and, for every $\varepsilon>0$, there exist $T=T(\varepsilon) \geq 0$ and $\rho=\rho(\varepsilon)>0$ such that if $\bar{x}:[\gamma, v] \rightarrow B_{c}, t_{0}-r \leq \gamma<v<+\infty$, is a function of bounded variation in $[\gamma, v]$ such that

$$
\|\bar{x}(\gamma)\|<\delta_{0}
$$

and

$$
\operatorname{var}_{\gamma}^{v}\left(\bar{x}(s)-\int_{\gamma}^{s} D F(\bar{x}(\tau), t)\right)<\rho,
$$

then

$$
\|\bar{x}(t)\|<\varepsilon \quad \text { for } \quad t \in[\gamma, v] \cap[\gamma+T,+\infty) \quad \text { and } \quad \gamma \geq t_{0}-r .
$$


Definition 3.3. The solution $x \equiv 0$ of (3.1) is called variationally asymptotically stable if it is variationally stable and variationally attracting.

For Definitions 3.1-3.3, it should be noted that if $\bar{x}:[\gamma, v] \rightarrow X$ is a solution of (3.1) then

(a) $\bar{x}$ is of bounded variation on $[\gamma, v]$ and

(b) $\operatorname{var}_{\gamma}^{v}\left(\bar{x}(s)-\int_{\gamma}^{s} D F(\bar{x}(\tau), t)\right)=0$.

The conditions in Definition 3.1 also mean that the function $\bar{x}$ of bounded variation is close, in the variation norm $\|\bar{x}(\gamma)\|+\operatorname{var}\left(\bar{x}(s)-\int_{\gamma}^{s} D F(\bar{x}(\tau), t)\right)$, to the solution $x \equiv 0$ of (3.1).

In addition to the generalized differential equation (3.1), let us consider the perturbed generalized equation

$$
\frac{d x}{d \tau}=D[F(x, t)+\bar{P}(t)]
$$

where $\bar{P} \in B V^{-}\left(\left[t_{0}-r, \infty\right), X\right)$. It is easy to verify that, for the function $G(x, t)=F(x, t)+\bar{P}(t)$, we have $G \in \mathcal{F}\left(\Omega, h_{\bar{P}}\right)$, where $h_{\bar{P}}(t)=h(t)+\operatorname{var}_{-r}^{t} \bar{P}$. Therefore, the solutions of (3.2) have good properties (existence, uniqueness, etc.; see, e.g., Theorem 2.1).

We now present some other definitions.

Definition 3.4. The solution $x \equiv 0$ of (3.1) is called stable with respect to perturbations if, for every $\varepsilon>0$, there exists $\delta=\delta(\varepsilon)>0$ such that if $\left\|x_{0}\right\|<\delta$ and $\bar{P} \in B V^{-}([\gamma, v], X)$ is continuous from the left with $\operatorname{var}_{\gamma}^{v} \bar{P}<\delta$, then

$$
\left\|x\left(t, \gamma, x_{0}\right)\right\|<\varepsilon \quad \text { for every } t \in[\gamma, v]
$$

where $x\left(t, \gamma, x_{0}\right)$ is a solution of the perturbed generalized equation (3.2) with $x\left(\gamma, \gamma, x_{0}\right)=x_{0}$ and $[\gamma, v] \subset$ $\left[t_{0}-r,+\infty\right)$.

Definition 3.5. The solution $x \equiv 0$ of (3.1) is called attracting with respect to perturbations if there is $\widetilde{\delta}>0$ and, for every $\varepsilon>0$, there exist $T=T(\varepsilon) \geq 0$ and $\rho=\rho(\varepsilon)>0$ such that if

$$
\left\|x_{0}\right\|<\widetilde{\delta} \quad \text { and } \quad \operatorname{var}_{\gamma}^{v} \bar{P}<\rho
$$

with $\bar{P} \in B V^{-}([\gamma, v], X)$, then

$$
\left\|x\left(t, \gamma, x_{0}\right)\right\|<\varepsilon \quad \text { for all } t \geq \gamma+T, \quad t \in[\gamma, v]
$$

where $x\left(t, \gamma, x_{0}\right)$ is a solution of the perturbed generalized equation (3.2) with $x\left(\gamma, \gamma, x_{0}\right)=x_{0}$ and $[\gamma, v] \subset$ $\left[t_{0}-r,+\infty\right)$.

Definition 3.6. The solution $x \equiv 0$ of (3.1) is called asymptotically stable with respect to perturbations if it is both stable and attracting with respect to perturbations.

It turns out that the corresponding definitions presented above are equivalent. Indeed, we have the following result: 
Proposition 3.1. The following statements are true:

(i) The solution $x \equiv 0$ of (3.1) is variationally stable if and only if it is stable with respect to perturbations.

(ii) The solution $x \equiv 0$ of (3.1) is variationally attracting if and only if it is attracting with respect to perturbations.

(iii) The solution $x \equiv 0$ of (3.1) is variationally asymptotically stable if and only if it is asymptotically stable with respect to perturbations.

Proof. Let us prove (i). Assume that the solution $x \equiv 0$ of (3.1) is variationally stable. Let, for $\varepsilon>0$, the quantity $\delta>0$ be given according to Definition 3.4. Then, for the solution $x(t)=x\left(t, \gamma, x_{0}\right)$ of the perturbed generalized equation (3.2) on $[\gamma, v]$, we have $\|x(\gamma)\|=\left\|x\left(\gamma, \gamma, x_{0}\right)\right\|<\delta$, and, for any $s_{1}, s_{2} \in[\gamma, v]$, we get

$$
x\left(s_{2}\right)-x\left(s_{1}\right)=\int_{s_{1}}^{s_{2}} D F(x(\tau), t)+\bar{P}\left(s_{2}\right)-\bar{P}\left(s_{1}\right),
$$

i.e.,

$$
x\left(s_{2}\right)-\int_{\gamma}^{s_{2}} D F(x(\tau), t)-\left(x\left(s_{1}\right)-\int_{\gamma}^{s_{1}} D F(x(\tau), t)\right)=\bar{P}\left(s_{2}\right)-\bar{P}\left(s_{1}\right),
$$

whence

$$
\operatorname{var}_{\gamma}^{v}\left(x(s)-\int_{\gamma}^{s} D F(x(\tau), t)\right)=\operatorname{var}_{\gamma}^{v} \bar{P}<\delta
$$

Therefore, the variational stability implies that

$$
\|x(t)\|=\left\|x\left(t, \gamma, x_{0}\right)\right\|<\varepsilon \text { for } t \in[\gamma, v]
$$

and the trivial solution of (3.1) is stable with respect to perturbations.

Conversely, if the solution $x \equiv 0$ of (3.1) is stable with respect to perturbations, we take $\bar{x}:[\gamma, v] \rightarrow B_{c}$, $-r \leq \gamma<v<+\infty$, a function of bounded variation on $[\gamma, v]$ such that $\|\bar{x}(\gamma)\|<\delta$ and

$$
\operatorname{var}_{\gamma}^{v}\left(\bar{x}(s)-\int_{\gamma}^{s} D F(\bar{x}(\tau), t)\right)<\delta
$$

where $\delta>0$ corresponds to some $\varepsilon>0$ from Definition 3.4.

For $s \in[\gamma, v]$, let $\bar{P}(s)=\bar{x}(s)-\int_{\gamma}^{s} D F(\bar{x}(\tau), t)$. Then, for $s_{1}, s_{2} \in[\gamma, v]$, we have

$$
\bar{P}\left(s_{2}\right)-\bar{P}\left(s_{1}\right)=\bar{x}\left(s_{2}\right)-\bar{x}\left(s_{1}\right)-\int_{s_{1}}^{s_{2}} D F(\bar{x}(\tau), t) .
$$


Hence,

$$
\bar{x}\left(s_{2}\right)-\bar{x}\left(s_{1}\right)=\int_{s_{1}}^{s_{2}} D F(\bar{x}(\tau), t)+\bar{P}\left(s_{2}\right)-\bar{P}\left(s_{1}\right), \quad s_{1}, s_{2} \in[\gamma, v],
$$

which means that $\bar{x}$ is a solution of (3.2) in $[\gamma, v]$. Furthermore, $\operatorname{var}_{\gamma}^{v} \bar{P}<\delta, \bar{P}$ is left continuous, and $\|\bar{x}(\gamma)\|=\left\|\bar{x}\left(\gamma, \gamma, x_{0}\right)\right\|=\|\bar{P}(\gamma)\|<\delta$. Therefore, the stability with respect to perturbations implies that $\|\bar{x}(t)\|=\left\|\bar{x}\left(t, \gamma, x_{0}\right)\right\|<\varepsilon$ for all $t \in[\gamma, v]$, and this means that the solution $x \equiv 0$ of (3.1) is variationally stable.

Coming to the attractive part in item (ii), assume first that the solution $x \equiv 0$ of (3.1) is variationally attracting. Then there is $\delta_{0}>0$ and, for every $\varepsilon>0$, there exist $T=T(\varepsilon) \geq 0$ and $\rho=\rho(\varepsilon)>0$ such that if $\bar{x}:[\gamma, v] \rightarrow B_{c}, \quad-r \leq \gamma<v<+\infty$, is a function of bounded variation in $[\gamma, v]$ such that $\|\bar{x}(\gamma)\|<\delta_{0}$ and

$$
\operatorname{var}_{\gamma}^{v}\left(\bar{x}(s)-\int_{\gamma}^{s} D F(\bar{x}(\tau), t)\right)<\rho
$$

then

$$
\|\bar{x}(t)\|<\varepsilon, \quad t \in[\gamma, v] \cap[\gamma+T,+\infty), \quad \gamma \geq-r
$$

If $\left\|x_{0}\right\|<\widetilde{\delta}$ and $\bar{P} \in B V^{-}([\gamma, v], X)$ is such that $\operatorname{var}_{\gamma}^{v} \bar{P}<\rho$, then denote by $x(t)=x\left(t, \gamma, x_{0}\right)$ the solution of the perturbed generalized equation (3.2) satisfying $x\left(\gamma, \gamma, x_{0}\right)=x_{0}$. It follows that $\|x(\gamma)\|<\widetilde{\delta}$, and we have

$$
\operatorname{var}_{\gamma}^{v}\left(x(s)-\int_{\gamma}^{s} D F(x(\tau), t)\right)=\operatorname{var}_{\gamma}^{v} \bar{P}<\delta
$$

Hence, by Definition 3.2, we get

$$
\left\|x\left(t, \gamma, x_{0}\right)\right\|=\|x(t)\|<\varepsilon \quad \text { for all } t \geq \gamma+T, \quad t \in[\gamma, v],
$$

i.e., the solution $x \equiv 0$ of (3.1) is attracting with respect to perturbations.

Conversely, if the solution $x \equiv 0$ of (3.1) is attracting with respect to perturbations, assume that $\bar{x}:[\gamma, v] \rightarrow$ $B_{c},-r \leq \gamma<v<+\infty$, is a left continuous function of bounded variation in $[\gamma, v]$ such that $\|\bar{x}(\gamma)\|<\delta_{0}$ and

$$
\operatorname{var}_{\gamma}^{v}\left(\bar{x}(s)-\int_{\gamma}^{s} D F(\bar{x}(\tau), t)\right)<\rho
$$

As in the previous part of the proof, it is easy to see that $\bar{x}(t)$ is a solution of (3.2) on $[\gamma, v]$, where $\bar{P}(s)=$ $\bar{x}(s)-\int_{\gamma}^{s} D F(\bar{x}(\tau), t)$ for $s \in[\gamma, v]$. This function $\bar{P}$ belongs to $B V^{-}([\gamma, v], X)$, and there exists $\delta_{0}>0$ and, for every $\varepsilon>0$, there exist $T=T(\varepsilon) \geq 0$ and $\rho=\rho(\varepsilon)>0$ such that $\operatorname{var}_{\gamma}^{v} \bar{P}<\rho$. Definition 3.5 now yields 


$$
\|\bar{x}(t)\|<\varepsilon, \quad t \in[\gamma, v] \cap[\gamma+T,+\infty), \quad \gamma \geq-r
$$

which means that we have the variational attractivity of the trivial solution of (3.1).

Item (iii) follows from (i) and (ii), and we complete the proof.

\section{Stability Relations between Equations}

Consider the retarded system (1.1). Let $G_{1} \subset G\left(\left[t_{0}-r,+\infty\right), \mathbb{R}^{n}\right)$ be defined as in the beginning of the paper.

We assume that $f(\phi, t): G_{1} \times\left[t_{0},+\infty\right) \rightarrow \mathbb{R}^{n}$ is such that, for every $y \in G_{1}$, the mapping $t \mapsto f\left(y_{t}, t\right)$ belongs to $L_{1}\left(\left[t_{0}-r,+\infty\right), \mathbb{R}^{n}\right)$ and conditions (A) and (B) are satisfied. Assume, in addition, that $f(0, t)=0$ for every $t \in\left[t_{0},+\infty\right)$. Thus, $y \equiv 0$ is a solution of $(1.1)$ in $\left[t_{0}-r,+\infty\right)$.

For $y \in G_{1}$ and $t \in\left[t_{0}-r,+\infty\right)$, we define $F(y, t)$ as in (2.6). Then

$$
F: G_{1} \times\left[t_{0}-r,+\infty\right) \rightarrow C\left(\left[t_{0}-r,+\infty\right), \mathbb{R}^{n}\right)
$$

and, by definition, we have $F(0, t)=0$ for all $t \in\left[t_{0}-r,+\infty\right)$. Then $x \equiv 0$ is a solution of the generalized differential equation

$$
\frac{d x}{d \tau}=D F(x, t)
$$

in $\left[t_{0}-r,+\infty\right)$.

By the results from Proposition 2.2, there is a well-described one-to-one correspondence between solutions of Eqs. (1.1) and (4.1) with $F$ given by (2.6).

We also consider the perturbed retarded equation (1.5) and, again by Proposition 2.2, its corresponding perturbed generalized equation

$$
\frac{d x}{d \tau}=D G(x, t)=D[F(x, t)+\bar{P}(t)]
$$

where $F$ is given by (2.6) and $\bar{P}$ is given by (2.7).

We have

$$
\bar{P}:\left[t_{0}-r,+\infty\right) \rightarrow G^{-}\left(\left[t_{0}-r,+\infty\right), \mathbb{R}^{n}\right)
$$

and, hence,

$$
G: G_{1} \times\left[t_{0}-r,+\infty\right) \rightarrow G^{-}\left(\left[t_{0}-r,+\infty\right), \mathbb{R}^{n}\right) .
$$

We can now present a result that relates the respective concepts of variational stability and variational attractivity of the trivial solution of the retarded equation (1.1) and the trivial solution of its corresponding generalized equation (4.1).

Theorem 4.1. The following statements are true:

(i) The solution $y \equiv 0$ of (1.1) is variationally stable if and only if the solution $x \equiv 0$ of (4.1) is variationally stable.

(ii) The solution $y \equiv 0$ of (1.1) is variationally attracting if and only if the solution $x \equiv 0$ of (4.1) is variationally attracting. 
(iii) The solution $y \equiv 0$ of (1.1) is variationally asymptotically stable if and only if the solution $x \equiv 0$ of (4.1) is variationally asymptotically stable.

Proof. We begin with proving (i). Assume that the trivial solution of (1.1) in $\left[t_{0}-r,+\infty\right)$ is variationally stable. Then, given $\varepsilon>0$, there exists $\delta=\delta(\varepsilon)>0$ such that if $\phi \in G^{-}\left([-r, 0], \mathbb{R}^{n}\right)$ is such that $\|\phi\|<\delta$ and $P(t)$ belongs to $B V^{-}\left(\left[t_{0}, t_{1}\right], \mathbb{R}^{n}\right)$ with $\operatorname{var}_{t_{0}}^{t_{1}} P<\delta$, then

$$
\left|y\left(t ; t_{0}, \phi\right)\right|<\frac{\varepsilon}{2}, \quad t \in\left[t_{0}, t_{1}\right]
$$

where $y\left(t ; t_{0}, \phi\right)$ is a solution of (1.5).

We want to prove that the trivial solution of the generalized equation (4.1) with $F$ given by (2.6) is stable with respect to perturbations. Then the result will follow by Proposition 3.1.

Assume that $\delta=\delta(\varepsilon)>0$ from Definition 1.6 is such that $\delta<\varepsilon / 2$. Let $x\left(t ; t_{0}, x_{0}\right)$ be a solution of the perturbed generalized equation (4.2) with $F$ given by (2.6), $\bar{P}$ given by (2.7), and $x\left(t_{0} ; t_{0}, x_{0}\right)=x_{0}$ and assume that $\left\|x_{0}\right\|<\delta$, where $x_{0} \in G^{-}\left(\left[t_{0}-r,+\infty\right), \mathbb{R}^{n}\right)$, and $\bar{P} \in B V^{-}\left(\left[t_{0}, t_{1}\right], \mathbb{R}^{n}\right)$ with $\operatorname{var}_{t_{0}}^{t_{1}} \bar{P}<\delta$.

We have $\left\|x\left(t_{0}\right)\right\|=\left\|x\left(t_{0} ; t_{0}, x_{0}\right)\right\|=\left\|x_{0}\right\|<\delta$, which means that $\sup _{\theta \in\left[t_{0}-r,+\infty\right)}\left|x\left(t_{0}\right)(\theta)\right|<\delta$ and, therefore, $\sup _{\theta \in\left[t_{0}-r, t_{0}\right]}\left|\phi\left(\theta-t_{0}\right)\right|<\delta$. Thus,

$$
\|\phi\|<\delta .
$$

Since $x$ is a solution of the perturbed generalized equation, we have

$$
x\left(s_{2}\right)-x\left(s_{1}\right)=\int_{s_{1}}^{s_{2}} D[F(x(\tau), t)+\bar{P}(t)]=\int_{s_{1}}^{s_{2}} D F(x(\tau), t)+\bar{P}\left(s_{2}\right)-\bar{P}\left(s_{1}\right)
$$

for $s_{1}, s_{2} \in\left[t_{0}, t_{1}\right]$.

Therefore,

$$
x\left(s_{2}\right)-\int_{t_{0}}^{s_{2}} D F(x(\tau), t)-x\left(s_{1}\right)+\int_{t_{0}}^{s_{1}} D F(x(\tau), t)=\bar{P}\left(s_{2}\right)-\bar{P}\left(s_{1}\right) .
$$

Hence,

$$
\operatorname{var}_{t_{0}}^{t_{1}}\left(x(s)-\int_{t_{0}}^{s} D F(x(\tau), t)\right)=\operatorname{var}_{t_{0}}^{t_{1}} \bar{P}<\delta .
$$

Thus, by the variational stability of the trivial solution of (1.1), we have $|y(t)|<\varepsilon / 2$ for all $t \in\left[t_{0}, t_{1}\right]$.

Finally, we get

$$
\|x(t)\|=\sup _{\theta \in\left[t_{0}-r,+\infty\right)}|x(t)(\theta)|=\sup _{\theta \in\left[t_{0}-r, t\right]}|y(\theta)| \leq\|\phi\|+\sup _{\theta \in\left[t_{0}, t\right]}|y(\theta)| \leq \delta+\frac{\varepsilon}{2}<\varepsilon
$$

and we have the sufficiency of item (i).

Using (i) from Proposition 3.1, we now assume that the trivial solution of (4.1) is stable with respect to perturbations. Thus, given $\varepsilon>0$, let $\delta=\delta(\varepsilon)>0$ be the quantity from Definition 3.4. 
Let $y\left(t ; t_{0}, \phi\right)$ be a solution of the perturbed retarded equation (1.5). Suppose that $\|\phi\|<\delta$ and $P \in$ $B V^{-}\left(\left[t_{0}, t_{1}\right], \mathbb{R}^{n}\right)$ with $\operatorname{var}_{t_{0}}^{t_{1}} P<\delta$. We want to prove that $y \equiv 0$ is variationally stable, i.e., $\left|y\left(t ; t_{0}, \phi\right)\right|<\varepsilon$, $t \in\left[t_{0}, t_{1}\right]$. Then the converse of item (i) (necessity) will follow by Proposition 3.1.

Let $x\left(t ; t_{0}, x_{0}\right)$ be the solution of the perturbed generalized equation (4.2) with $F$ given by (2.6) and $\bar{P}$ given by (2.7), i.e., $x$ is the solution corresponding to $y$ obtained according to Proposition 2.2. We have $\operatorname{var}_{t_{0}}^{t_{1}} x \leq \operatorname{var}_{t_{0}}^{t_{1}} y$ (see the comments after Proposition 2.2) and, analogously, $\operatorname{var}_{t_{0}}^{t_{1}} \bar{P} \leq \operatorname{var}_{t_{0}}^{t_{1}} P<\delta$. Thus, it follows from the stability with respect to perturbations of the trivial solution of (4.1) that $\|x(t)\|<\varepsilon$, i.e.,

$$
\sup _{\theta \in\left[t_{0}-r,+\infty\right)}|x(t)(\theta)|<\varepsilon .
$$

Therefore, the relation in Proposition 2.2 implies that

$$
\sup _{\theta \in\left[t_{0}-r, t\right]}|y(\theta)|<\varepsilon, \quad t \in\left[t_{0}, t_{1}\right] .
$$

In particular,

$$
\sup _{\theta \in\left[t_{0}, t_{1}\right]}|y(\theta)| \leq \sup _{\theta \in\left[t_{0}-r, t_{1}\right]}|y(\theta)|<\varepsilon
$$

We now prove (ii).

First, suppose that the trivial solution of the retarded equation (1.1) is variationally attracting. Thus, there exists $\delta_{0}>0$, and, for every $\varepsilon>0$, let $T=T(\varepsilon) \geq 0$ and $\rho=\rho(\varepsilon)>0$ be from Definition 1.7.

Let $x\left(t ; t_{0}, x_{0}\right)$ be a solution of the perturbed generalized equation (4.2) with $F$ given by (2.6) and $\bar{P}$ given by (2.7) and let $y\left(t ; t_{0}, \phi\right)$ be the solution of the perturbed retarded equation (1.5) obtained from $x$ according to Proposition 2.2.

Let $\delta>0$ be such that $\left\|x_{0}\right\|<\delta$ and suppose that $\bar{P} \in B V^{-}\left(\left[t_{0}, t_{1}\right], \mathbb{R}^{n}\right)$ with $\operatorname{var}_{t_{0}}^{t_{1}} \bar{P}<\rho$. We can assume, without loss of generality, that $\delta<\min \left\{\delta_{0}, \rho, \varepsilon / 2\right\}$. Then

$$
\left\|x\left(t_{0}\right)\right\|=\left\|x_{0}\right\|<\delta_{0}
$$

and

$$
\operatorname{var}_{t_{0}}^{t_{1}}\left(x(s)-\int_{t_{0}}^{s} D F(x(\tau), t)\right)=\operatorname{var}_{t_{0}}^{t_{1}} \bar{P}<\rho .
$$

However, the variational attractivity of the trivial solution of (1.1) yields

$$
\left|y\left(t ; t_{0}, \phi\right)\right|=|y(t)|<\frac{\varepsilon}{2}, \quad t \geq t_{0}+T, \quad t \in\left[t_{0}, t_{1}\right] .
$$

Then, taking $\delta<\varepsilon / 2$, we obtain

$$
\|x(t)\|=\sup _{\theta \in\left[t_{0}-r,+\infty\right)}|x(t)(\theta)|=\sup _{\theta \in\left[t_{0}-r, t\right]}|y(\theta)| \leq\|\phi\|+\sup _{\theta \in\left[t_{0}, t\right]}|y(\theta)|<\left\|x_{0}\right\|+\frac{\varepsilon}{2}<\varepsilon
$$


for $t \geq t_{0}+T, t \in\left[t_{0}, t_{1}\right]$, where we have applied the relations of Proposition 2.2 to get the second equality and $\|\phi\|=\left\|x_{0}\right\|$, since

$$
\left\|x_{0}\right\|=\left\|x\left(t_{0}\right)\right\|=\sup _{\theta \in\left[t_{0}-r,+\infty\right)}|x(t)(\theta)|=\sup _{\theta \in\left[t_{0}-r, t_{0}\right]}\left|\phi\left(\theta-t_{0}\right)\right|=\|\phi\| .
$$

Thus,

$$
\left\|x\left(t ; t_{0}, x_{0}\right)\right\|=\|x(t)\|<\varepsilon, \quad t \geq t_{0}+T, \quad t \in\left[t_{0}, t_{1}\right],
$$

and, hence, $x$ is attracting with respect to perturbations. The sufficiency of (ii) follows then by Proposition 3.1.

We now prove the converse of item (ii). Suppose that the trivial solution of the generalized equation (4.1) is attracting with respect to perturbations. Then there exists $\delta_{0}>0$, and, given $\varepsilon>0$, let $T=T(\varepsilon) \geq 0$ and $\rho=\rho(\varepsilon)>0$ be from Definition 3.5.

Let $y\left(t ; t_{0}, \phi\right)$ be a solution of the perturbed retarded equation (1.3), or, equivalently, of Eq. (1.5) with $P(t)=$ $\int_{t_{0}}^{t} p(s) d s, t \geq t_{0}$. Suppose that $\|\phi\|<\delta_{0}$ and $P \in B V^{-}\left(\left[t_{0}, t_{1}\right], \mathbb{R}^{n}\right)$ with $\operatorname{var}_{t_{0}}^{t_{1}} P<\rho$.

By Proposition 2.2, it follows that $\left\|x_{0}\right\|=\|\phi\|<\delta_{0}$. Also, for $\bar{P}$ given by (2.7), we have $\operatorname{var}_{t_{0}}^{t_{1}} \bar{P} \leq$ $\operatorname{var}_{t_{0}}^{t_{1}} P<\rho$ (see the comments after Proposition 2.2). Therefore, the attractivity with respect to perturbations of the trivial solution of (4.1) yields

$$
\|x(t)\|=\left\|x\left(t ; t_{0}, x_{0}\right)\right\|<\varepsilon, \quad t \geq t_{0}+T, \quad t \in\left[t_{0}, t_{1}\right] .
$$

Therefore, for $t \geq t_{0}+T, t \in\left[t_{0}, t_{1}\right]$, by Proposition 2.2 we have

$$
|y(t)|=\left|y\left(t ; t_{0}, \phi\right)\right|=|x(t)(t)| \leq\|x(t)\|<\varepsilon .
$$

Assertion (iii) follows from (i) and (ii) and from Proposition 3.1.

\section{Converse Lyapunov Theorems}

In the book [7] and in [4], direct Lyapunov-type theorems for stability of a solution of a GODE are given. In [3], they are used for Eq. (1.1).

Converse Lyapunov theorems are an interesting topic, and we present them shortly in this concluding section of the paper.

In order to obtain converse Lyapunov theorems for Eq. (1.1), we need the following results, borrowed from [7] or [4], for the generalized differential equation (4.1):

Let us consider the general case where $\Omega=B_{c} \times\left[t_{0}-r, \infty\right), B_{c}=\{y \in X ;\|y\|<c\}, c>0$, and $X$ is a Banach space. Suppose that $F: \Omega \rightarrow X$ is such that $F \in \mathcal{F}(\Omega, h)$ and $F(0, t)-F(0, s)=0$ for $t, s \in\left[t_{0}-r,+\infty\right)$ and consider the generalized differential equation

$$
\frac{d x}{d \tau}=D F(x, t)
$$

The following two results are Theorems 10.23 and 10.24, respectively, from [7]. They can also be found in [4]. 
Theorem 5.1. If the trivial solution $x \equiv 0$ of the generalized differential equation (5.1) is variationally stable, then, for every $0<a<c$, there exists a function $V:\left[t_{0}-r,+\infty\right) \times B_{a} \rightarrow \mathbb{R}$, where $B_{a}=\{y \in$ $X ;\|y\|<a\}$, such that, for every $x \in B_{a}$, the function $V(\cdot, x)$ belongs to $B V^{-}\left(\left[t_{0}-r,+\infty\right), \mathbb{R}\right)$ and the following conditions are satisfied:

(i) $V(t, 0)=0, \quad t \in\left[t_{0}-r,+\infty\right)$;

(ii) $|V(t, z)-V(t, y)| \leq\|z-y\|, t \in\left[t_{0}-r,+\infty\right), z, y \in B_{a}$;

(iii) $V$ is positive definite along every solution $x(t)$ of the generalized equation (5.1), i.e., there is a function $b:[0,+\infty) \rightarrow \mathbb{R}$ of Hahn class such that

$$
V(t, x(t)) \geq b(\|x(t)\|), \quad(t, x(t)) \in\left[t_{0}-r,+\infty\right) \times B_{a}
$$

(iv) for all solutions $x(t)$ of (5.1),

$$
\dot{V}(t, x(t))=\limsup _{\eta \rightarrow 0_{+}} \frac{V(t+\eta, x(t+\eta))-V(t, x(t))}{\eta} \leq 0,
$$

i.e., the right derivative of $V$ along every solution $x(t)$ of (5.1) is nonpositive.

Theorem 5.2. If the trivial solution $x \equiv 0$ of the generalized differential equation (5.1) is variationally asymptotically stable, then, for every $0<a<c$, there exists a function $V:\left[t_{0}-r,+\infty\right) \times B_{a} \rightarrow \mathbb{R}$ such that, for every $x \in B_{a}$, the function $V(\cdot, x)$ belongs to $B V^{-}\left(\left[t_{0}-r,+\infty\right), \mathbb{R}\right)$ and the following conditions are satisfied:

(i) $V(t, 0)=0, \quad t \in\left[t_{0}-r,+\infty\right)$;

(ii) $|V(t, z)-V(t, y)| \leq\|z-y\|, \quad t \in\left[t_{0}-r,+\infty\right), z, y \in B_{a}$;

(iii) $V$ is positive definite along every solution $x(t)$ of the generalized equation (5.1), i.e., there is a function $b:[0,+\infty) \rightarrow \mathbb{R}$ of Hahn class such that

$$
V(t, x(t)) \geq b(\|x(t)\|), \quad(t, x(t)) \in\left[t_{0}-r,+\infty\right) \times B_{a}
$$

(iv) for all solutions $x(s)$ of (5.1) defined for $s \geq t$, where $x(t)=z \in B_{a}$, the following relation is true:

$$
\dot{V}(t, x(t))=\limsup _{\eta \rightarrow 0_{+}} \frac{V(t+\eta, x(t+\eta))-V(t, x(t))}{\eta} \leq V(t, z) .
$$

Now let us consider a more specialized equation (4.1) with $F$ given by (2.6) that corresponds to the retarded system (1.1). We consider $X=G^{-}\left(\left[t_{0}-r,+\infty\right), \mathbb{R}^{n}\right)$. As in [3], we need to relate a Lyapunov functional for (4.1) to a Lyapunov functional for (1.1)

Let $y:[\gamma, v] \rightarrow \mathbb{R}^{n}$ be a solution of Eq. (1.1) on $[\gamma, v] \subset\left[t_{0}-r,+\infty\right),[\gamma, v] \ni t_{0}$, such that $y_{t}=\psi$ for a given $t \geq t_{0}$, i.e., $\psi \in G^{-}\left([-r, 0], \mathbb{R}^{n}\right)$ and

$$
\psi(\theta)=y_{t}(\theta)=y(t+\theta)=y(t)-\int_{[t+\theta, t]} f\left(y_{s}, s\right) d s
$$


for almost every $\theta \in[-r, 0]$. In this case, we write $y_{t+\eta}=y_{t+\eta}(t, \psi)$ for every $\eta \geq 0$. If $U:\left[t_{0}-r,+\infty\right) \times$ $G^{-}\left([-r, 0], \mathbb{R}^{n}\right) \rightarrow \mathbb{R}$, then we define

$$
D^{+} U(t, \psi)=\limsup _{\eta \rightarrow 0_{+}} \frac{U\left(t+\eta, y_{t+\eta}(t, \psi)\right)-U\left(t, y_{t}(t, \psi)\right)}{\eta}
$$

for $t \geq t_{0}$.

Let $x$ be a solution of the generalized equation (4.1) on the interval $[\gamma, v] \subset\left[t_{0}-r,+\infty\right),[\gamma, v] \ni t_{0}$, with initial condition $x\left(t_{0}\right)=x_{0}$, where

$$
x\left(t_{0}\right)(\vartheta)=\left\{\begin{array}{lll}
\phi\left(\vartheta-t_{0}\right) & \text { for } & \vartheta \in\left[t_{0}-r, t_{0}\right], \\
x\left(t_{0}\right)\left(t_{0}\right) & \text { for } \quad \vartheta \in\left[t_{0},+\infty\right) .
\end{array}\right.
$$

Then $x(t)(t+\theta)=y(t+\theta)$ for all $t \in\left[t_{0}-r,+\infty\right)$ and $\theta \in[-r, 0]$, and, hence, $(x(t))_{t}=y_{t}$ for all $t$.

On the other hand, given $x(t) \in G^{-}\left(\left[t_{0}-r,+\infty\right), \mathbb{R}^{n}\right)$, since $x$ is locally of bounded variation, we can consider $x(t)$ as a solution on $[\gamma, v] \subset\left[t_{0}-r,+\infty\right),[\gamma, v] \ni t_{0}$, of the generalized equation (4.1) with initial condition $x\left(t_{0}\right)=x_{0}$ given by (5.2). Then Proposition 2.2 implies that we can find a solution $y\left(t ; t_{0}, \phi\right)$ of $(1.1)$ by using the solution $x\left(t ; t_{0}, x_{0}\right)$ of (4.1). Let $(x(t))_{t}=\psi$. In this case, we write $x_{\psi}(t)$ instead of $x(t)$, and we have $y_{t}=\psi$.

Consequently, $\left(t, x_{\psi}(t)\right) \mapsto\left(t, y_{t}(t, \psi)\right)$ is a one-to-one mapping, and we can define $V:\left[t_{0}-r,+\infty\right) \times$ $G^{-}\left(\left[t_{0}-r,+\infty\right), \mathbb{R}^{n}\right) \rightarrow \mathbb{R}$ by

$$
V\left(t, x_{\psi}(t)\right)=U\left(t, y_{t}(t, \psi)\right), \quad t \geq t_{0}
$$

Then we have

$$
D^{+} U(t, \psi)=\limsup _{\eta \rightarrow 0_{+}} \frac{V\left(t+\eta, x_{\psi}(t+\eta)\right)-V\left(t, x_{\psi}(t)\right)}{\eta}
$$

for all $t \geq t_{0}$. We write $\dot{U}\left(t, y_{t}\right)=D^{+} U\left(t, y_{t}\right)$.

With the notation above, we can now present converse Lyapunov results for Eq. (1.1).

Theorem 5.3. If the trivial solution $y \equiv 0$ of the retarded differential equation (1.1) is variationally stable, then, for every $0<a<c$, there exists a function $U:\left[t_{0}-r,+\infty\right) \times E_{a} \rightarrow \mathbb{R}$, where $E_{a}=\left\{\psi \in G^{-}\left([-r, 0], \mathbb{R}^{n}\right) ;\|\psi\|<a\right\}$, such that, for every $x \in E_{a}$, the function $U(\cdot, \psi)$ belongs to $B V^{-}\left(\left[t_{0}-r,+\infty\right), \mathbb{R}\right)$ and the following conditions are satisfied:

(i) $U(t, 0)=0, \quad t \in\left[t_{0}-r,+\infty\right)$;

(ii) $|U(t, \psi)-U(t, \bar{\psi})| \leq\|\psi-\bar{\psi}\|, t \in\left[t_{0}-r,+\infty\right), \psi, \bar{\psi} \in E_{a}$;

(iii) $U$ is positive definite along every solution $y(t)$ of the retarded equation (1.1), i.e., there is a function $b:[0,+\infty) \rightarrow \mathbb{R}$ of Hahn class such that

$$
U\left(t, y_{t}\right) \geq b\left(\left\|y_{t}\right\|\right), \quad\left(t, y_{t}\right) \in\left[t_{0}-r,+\infty\right) \times E_{a} ;
$$


(iv) for all solutions $y(t)$ of (1.1),

$$
\dot{U}\left(t, y_{t}\right)=\limsup _{\eta \rightarrow 0_{+}} \frac{\left.U\left(t+\eta, y_{t+\eta}\right)-V\left(t, y_{t}\right)\right)}{\eta} \leq 0
$$

i.e., the right derivative of $U$ along every solution $y(t)$ of $(1.1)$ is nonpositive.

Proof. If the trivial solution of (1.1) is variationally stable, then, by Theorem 4.1, the trivial solution of the generalized equation (4.1) with $F$ given by (2.6) and $\bar{P}$ given by (2.7) is also variationally stable. Then, by Theorem 5.1, there exists a function $V$ satisfying all conditions in that theorem. We define $U:\left[t_{0}-r,+\infty\right) \times$ $G^{-}\left([-r, 0], \mathbb{R}^{n}\right) \rightarrow \mathbb{R}$ by relation (5.3). Then, as in the proof of Theorem 4.3 in [3], $U$ has the properties above, and the proof is complete.

The proof of the next result follows as in the proof of Theorem 5.3, but with the use of Theorem 4.5 in [3] instead of Theorem 4.3 in [3].

Theorem 5.4. If the trivial solution $y \equiv 0$ of the retarded differential equation (1.1) is variationally asymptotically stable, then, for every $0<a<c$, there exists a function $U:\left[t_{0}-r,+\infty\right) \times E_{a} \rightarrow \mathbb{R}$ such that, for every $x \in B_{a}$, the function $U(\cdot, x)$ belongs to $B V^{-}\left(\left[t_{0}-r,+\infty\right), \mathbb{R}\right)$ and the following conditions are satisfied:

(i) $U(t, 0)=0, \quad t \in\left[t_{0}-r,+\infty\right)$;

(ii) $|U(t, \psi)-U(t, \bar{\psi})| \leq\|\psi-\bar{\psi}\|, t \in\left[t_{0}-r,+\infty\right), \psi, \bar{\psi} \in E_{a}$;

(iii) $U$ is positive definite along every solution $y(t)$ of the retarded equation (1.1), i.e., there is a function $b:[0,+\infty) \rightarrow \mathbb{R}$ of Hahn class such that

$$
U\left(t, y_{t}\right) \geq b\left(\left\|y_{t}\right\|\right), \quad\left(t, y_{t}\right) \in\left[t_{0}-r,+\infty\right) \times E_{a} ;
$$

(iv) for all solutions $y(s)$ of (1.1) defined for $s \geq t$, where $y(t)=\psi \in E_{a}$, the following relation is true:

$$
\dot{U}\left(t, y_{t}\right)=\limsup _{\eta \rightarrow 0_{+}} \frac{U\left(t+\eta, y_{t+\eta}\right)-U\left(t, y_{t}\right)}{\eta} \leq U(t, \psi) .
$$

This work was supported by the Grant Agency of the Academy of Sciences of the Czech Republic (Grant No. IAA100190702).

\section{REFERENCES}

1. J. K. Hale and S. M. Verduyn Lunel, Introduction to Functional Differential Equations, Springer, New York (1993).

2. A. Halanay, Differential Equations: Stability, Oscillations, Time Lags, Academic Press, New York (1966).

3. M. Federson and Š. Schwabik, A New Approach to Impulsive Retarded Differential Equations: Stability Results, Preprint (2002).

4. Š. Schwabik, "Variational stability for generalized ordinary differential equations," Čas. Pěstov. Mat., 109, No. 4, 389-420 (1984).

5. A. M. Samoilenko and N. A. Perestyuk, Impulsive Differential Equations, World Scientific, Singapore (1995).

6. M. Federson and Š. Schwabik, "Generalized ODEs approach to impulsive retarded differential equations," Different. Integral Equat., 19, No. 11, 1201-1234 (2006).

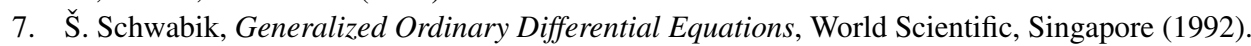

8. C. Imaz and Z. Vorel, "Generalized ordinary differential equations in Banach spaces and applications to functional equations," Bol. Soc. Mat. Mexic., 11, 47-59 (1966).

9. F. Oliva and Z. Vorel, "Functional equations and generalized ordinary differential equations," Bol. Soc. Mat. Mexic., 11, 40-46 (1966). 